\title{
The Management of the Patient with Elevated Prostate Specific Antigen and a Negative Initial Prostate Biopsy
}

\author{
Vida $A O^{1,2^{\star}}$, Szöllősi $A^{1,2}$, Maier $A^{1,2}$, Boja $R M^{1}$, Mártha Orsolya ${ }^{1,2}$ \\ 1 Department of Urology, Mures County Hospital, Tîrgu Mureș \\ 2 University of Medicine and Pharmacy, Tîrgu Mureș
}

\begin{abstract}
Background: The prostate cancer (PCa) is the second most common cancer amongst men. An elevated prostate specific antigen (PSA) level can lead to PCa suspition, thus the confirmation has to be a histopathological one. However, not all increased PSA level means prostate cancer. Material and Method: This retrospective study presents the results of 422 ultrasound guided prostate biopsy (PB) performed at the Clinic of Urology Târgu- Mureș, between 2011-2012. Inclusion criteria: patients with at least one negative PB and an elevated value of PSA. Results: In a two year period, from the total of 422 PB (100\%), in $179(42.42 \%)$ patients the histopathological result was "negative". In 154 $(86 \%)$ of cases ultrasound guided biopsies were performed, mostly with an initial 6 core (98-54.74\% of patients). Average PSA level was $13.45 \mathrm{ng} / \mathrm{ml}(0.49-100 \mathrm{ng} / \mathrm{ml})$, the histological findings confirmed PCa in 52.58\% cases, normal prostatic tissue in 141 cases (78.77\%), atypical small acinar proliferation in 12 patients (6.70\%), prostate atrophy in 11 males (6.14\%), benign prostatic hyperplasia in 10 cases (5.59\%) and prostatitis in 5 cases (2.80\%). In 30 cases (16.75\%) rebiopsy was performed with a number of $10-12$ cores.

Conclusions: In order to increase PCa detection we should perform more cores during PB. In „negative” histopathological cases PSA should be monitorised and the biopsy should be repeated after 6 to 8 weeks from the initial biopsy. Patient's compliance plays a vital role in the follow-up of the procedure.
\end{abstract}

Keywords: prostate cancer, PSA, biopsy, atypical small acinar proliferation

Received: 15 July 2014 / Accepted: 04 November 2014

\section{Introduction}

Despite the fact that prostate cancer is mostly a slow-growing cancer, still, thousands of men die of the disease each year and prostate cancer represents at the present moment the second most common cause of cancer death in males [1]. It is well-known that the incidence of prostate cancer increases with age and the highest rates are found in the seventh decade.

The early and accurate detection of prostate cancer is critical for the appropriate management of the patients. In the detection of prostate cancer the first line of screening represents digital rectal examination and prostate specific antigen (PSA) determination [2-7]. Alone, the digital rectal examination has a low sensitivity and specificity in prostate cancer detection, having about $10 \%$ positive predictive value $[1,4]$. PSA is a kallikrein-like serine-protease, organ specific, produced almost exclusively by the epithelial cells of the prostate gland, it still remains the most used tumor marker for prostate cancer [2]. The standard PSA reference range in men's serum is $0,0-4,0 \mathrm{ng} / \mathrm{ml}$ [1]. The positive predictive value of digital rectal examination combined with determined serum PSA is about $30 \%$ in prostate cancer detection $[1,2]$.

Another useful diagnostic method in the prostate gland evaluation is the transrectal ultrasound of the prostate

* Correspondence to: Árpád Olivér Vida

E-mail: vidaarpad@yahoo.com
(TRUS). It is usually used when ultrasound guided prostatic biopsy is performed.

The indications for prostate biopsy according to European Association of Urology Guidelines (2013) are increased or rising PSA level, abnormal digital rectal examination finding. The first elevated PSA level should not prompt an immediate biopsy, it should be verified after a few weeks by the same assay under standardised conditions, in the same diagnostic laboratory, using the same methods [2].

In most cases the prostate biopsy and subsequent histological examination confirms the diagnosis of prostate cancer. There is no consensus about the optimum number of cores that should be taken [1]. There are some conditions which require even in the case of negative results a rebiopsy. Following the European Association of Urology Guideline (2014) the recommendations for repeat prostate biopsy are the rising or/and persistently elevated PSA, suspicious digital rectal examination, ASAP (Atypical small acinar proliferation), extensive HGPIN (prostatic intraepithelial neoplasia) $[2,5]$. With this retrospective study we would like to process and discuss our findings about the results of ultrasound guided prostate biopsies performed at the Clinic of Urology Târgu- Mureș, and correlate our results with the ones in the literature.

\section{Methods}

Our retrospective study includes 422 patients who have had at least one negative ultrasound guided prostate biopsy and 
an elevated PSA result in a two year period (2011-2012). The studied group was analyzed upon the following data: age, clinical examination (digital rectal examination), PSA, prostate volume, number of the cores taken, histopathological findings, personal pathological history, other urological intervention, and complications of the prostate biopsy. All patients were prepared before prostate biopsy with enema and they got antibiotic treatment after intervention. Most of the biopsies were performed under local anesthesia with lidocaine gel instillated intrarectal 5 minutes before the procedure. The transrectal biopsies were ultrasound guided, performed with a transrectal probe $(7.5 \mathrm{MHz})$, using an automatic Bioptic gun with an 18 Gauge needle.

\section{Results}

In a two year period (2011-2012) in the Clinic of Urology of Târgu- Mureș we've performed 422 prostate biopsies $(100 \%)$, from which in $179(42.42 \%)$ cases the result was a "negative" one. All the samples were evaluated by the same team of pathologists. The average age of our patients was 68.8 year (52-89 year).

The average PSA was $13.45 \mathrm{ng} / \mathrm{ml}(0.49-100 \mathrm{ng} / \mathrm{ml})$, with a median value of $9.94 \mathrm{ng} / \mathrm{ml}$. In 18 cases $(10 \%)$ we didn't find any results. Regarding the prostate volume the average was $45.8 \mathrm{~cm} 3(25-130 \mathrm{~cm} 3)$.

In most of the cases, more than $45 \%$ ( 82 cases) the clinical examination of the prostate was a negative one, we could not find any kind of suspicion for prostate cancer. The most frequent complications after PB that we found were: rectal bleeding, almost all the patients presented a minimal rectorrhagia less than 24 hour post biopsy, acute urinary retention, requiring bladder catheterization appeared in 94 cases $(52.5 \%)$. Two cases $(1.11 \%)$ were readmitted for fever $\left(>38 \mathrm{C}^{\circ}\right)$ and chills after a few days, these were resolved with association of antibiotic therapy, antipyretics, and anti-inflammatory drugs. From the point of view of associated diseases, grouped into urological and non-urological ones we found in most of the cases associated cardiological diseases like chronic cardiac insufficiency, hypertension, atrial fibrillation (54 cases; 30.16\%). From the urological group the most significant disorder was the chronic urinary retention due to the enlarged prostate gland in 103 cases $(57.54 \%)$.

In $14 \%$ ( 25 cases) of the patients prostate biopsy was performed under digital guidance, otherwise the remaining 154 patients the biopsy was ultrasound guided. The average of the chores taken was 6.32 (2-13 punctures/patient). The cancer detection rate was $57.6 \%$ at the initial biopsy (Table I).

In 30 cases $(16.75 \%)$ rebiopsy was performed, in 2 of this cases prostate cancer was found (at initial biopsy the histopathological finding was normal prostatic tissue and ASAP, the rebiopsy showed in both of the cases prostate cancer with 6 and 7 Gleason scores). In another case the $\mathrm{PCa}$ was diagnosed after transurethral prostate resection (TUR-P), Gleason score 7.
The histological findings of all our selected patients were: normal prostatic tissue in 141 cases $(78.77 \%)$, ASAP in 12 cases $(6.70 \%)$, prostate atrophy in 11 cases $(6.14 \%)$, benign prostatic hyperplasia in 10 cases $(5.59 \%)$ and prostatitis in 5 cases $(2.80 \%)$ (Table II).

We followed also the associated urological interventions post biopsy. In 29 cases (16.2\%) we performed a transurethral prostate resection or a suprapubic prostatectomy for obstructive purposes. Other urological procedures were: internal optic urethrotomy, TURBT (transurethral resection of bladder tumor), PNL (percutaneous nephrolithotomy), ureteroscopy with calculi manipulation, endo-vesical lithotripsy, penile amputation, in 15 cases $(8.37 \%)$ (Table III).

Table I. Distribution of the studied group by age, prostate size and initial number of biopsy cores

\begin{tabular}{lccc}
\hline Group & Median & Minimum & Maximum \\
\hline Age (years) & 69,00 & 52,00 & 89,00 \\
Number of cores & 6,00 & 2,00 & 13,00 \\
Prostate size $\left(\mathrm{cm}^{3}\right)$ & 42,00 & 25,00 & 130,00 \\
\hline
\end{tabular}

Table II: The pathological findings of the prostate biopsies

\begin{tabular}{lc}
\hline Histological findings & Number of cases \\
\hline Normal prostatic tissue & 141 \\
ASAP & 12 \\
Prostate atrophy & 11 \\
Benign prostatic hyperplasia & 10 \\
Prostatitis & 5 \\
\hline
\end{tabular}

Table III: Urological interventions after initial prostate biopsy

\begin{tabular}{lc}
\hline Interventions & Number \\
\hline Repeated prostate biopsy & 30 \\
TUR-P/suprapubic prostatectomy & 29 \\
Other & 15 \\
\hline
\end{tabular}

\section{Discussions}

The incidence of positive prostate biopsy rates for prostate cancer in our study is $52.58 \%$, this result broadly corresponds with the incidence rate found in other studies of this kind. This means that about half of initial prostate biopsies are followed by a negative result. How we could improve our results? Dates from the literature state that despite of increasing the initial core numbers the gape I prostate cancer diagnosis remains about 30\% [7]. Some studies describe up to 21 cores biopsy protocols, but this kind of extended protocols involve more complications [8], some of them important ones (haematospermia, haematuria, rectorrhagia, prostatitis, fever $\left(>38 \mathrm{C}^{\circ}\right)$, epididymitis, urinary retention $[2,5])$. Because of the post procedural potential complications, this invasive maneuver should be discussed with the patient, especially in cases when a rebiopsy is indicated. In order to increase the success rate, we introduced the Vienna nomogram protocol, which indicates the number of cores that should be sampled based on prostate size $\left(\mathrm{cm}^{3}\right)$ and group of age (year). 
Another question is what should we do if the patient who had an initial negative prostate biopsy still presents an elevated or a rising PSA level? The recommendation in these cases is to repeat the prostate biopsy and extend the number of cores according to European Association of Urology Guidelines (2014). Rebiopsy should be performed also in cases with suspicious digital rectal examination; atypical small acinar proliferation (ASAP); extensive (multiple biopsy sites) prostatic intraepithelial neoplasia (PIN) [2,9-10].

In every day practice the question: Should we rebiopsy every patient, with elevated PSA value without any sign of malignancy on digital rectal examination, is answered with Yes, if the patient has a life expectancy more than 10 years, or he had one of the conditions described above. One should take in consideration at least two values of PSA collected on different times, without any kind of manipulation on lower urinary tract before.

\section{Conclusions}

The younger the patient, the bigger the prostate more cores should be performed, the traditional sextant prostate biopsy is no longer valid. If exists any kind of suspicion for prostate cancer the biopsy should be repeated after 6 to 8 weeks from the initial biopsy. There are some conditions which require the rebiopsy such as: atypical small acinar proliferation, extensive intraepithelial neoplasia, rising or/ and persistently elevated PSA, suspicious digital rectal examination.

\section{Acknoledgment:}

This paper was partly supported by the Sectorial Operational Programme Human Resources Development, financed from the European Social Fund and by the Romanian Government under the contract number POSDRU 80641.

\section{References:}

1. May AW, Orlando ES: Prostate Cancer A Practical Guide, China, Saunders Elsevier, 2008.

2. Heidenreich A, Bastian PJ, Bellmunt J: European Association of Urology Guidelines on Prostate Cancer 2013, http://www.uroweb.org/gls/pdf/09_ Prostate_Cancer_LR.pdf

3. DjavanB: Prostate Biopsies and the Vienna Nomograms, European Urology, 2006;Suppl 5 500-510.

4. Nelson AW, Harvey RC, Parker RA, Department of Urology, Addenbrooke's Hospital, Cambridge, United Kingdom: Repeat prostate biopsy strategies after initial negative biopsy: meta-regression comparing cancer detection of transperineal, transrectal saturation and MRI guided biopsy. http://www. plosone.org/article/info\%3Adoi\%2F10.1371\%2Fjournal.pone.0057480

5. Ukimura O, Coleman J A, Emberton M: Contemporary Role of Systematic Prostate Biopsies: Indications, Techniques, and Implications for Patient Care. European urology 2012;63:214 - 230

6. Robert $J \mathrm{H}$, Stephen J F: 5-a reductase inhibitors and prostate cancer prevention: where do we turn now? BMC Medicine 2011;9:105

7. Werahera PN, Sullivan K, La Rosa FG, at all: Optimization of prostate cancer diagnosis by increasing the number of core biopsies based on gland volume. International journal of clinical and experimental pathology. 2012;5:892-899.

8. Van Renterghem K, Van Koeveringe G, Achten R, at all: A new algorithm in patients with elevated and/or rising prostate-specific antigen level, minor lower urinary tract symptoms, and negative multisite prostate biopsies. International urology and nephrology. 2010;42:29-38

9. Schoenfield L, Jones JS, Zippe CD, at all: The incidence of high-grade prostatic intraepithelial neoplasia and atypical glands suspicious for carcinoma on first-time saturation needle biopsy, and the subsequent risk of cancer. BJU international. 2007;99:770-774

10. Fadi Brimo, Robin T. Vollmer, at all: Outcome for Repeated Biopsy of the Prostate, Am J Clin Pathol 2007;128:648-652 